\title{
A POSSIBLE NEW SIGNATURE FOR HIGGS BOSONS*
}

\author{
H.E. HABER
}

Santa Cruz Institute for Particle Physics, University of California, Santa Cruz, CA 95064, USA

\section{KANI}

Randall Laboratory of Physics, University of Michigan, Ann Arbor, MI 48109, USA

\author{
G.L. KANE ${ }^{1}$ and M. QUIRÓS ${ }^{2}$ \\ CERN, Geneva, Switzerland
}

Received 29 May 1986

\begin{abstract}
If low-energy supersymmetry is realized in nature, and if the appropriate supersymmetric partner of the Higgs boson, $\tilde{\mathrm{h}}$, has a mass $m_{\tilde{\mathrm{h}}} \ll m_{\mathrm{Z}}$, then there is a decay chain $\mathrm{Z} \rightarrow \tilde{\mathrm{z}} \tilde{\mathrm{h}}$, followed by $\tilde{z} \rightarrow \mathrm{h} \tilde{h}$, which can be a significant part of all $\mathrm{Z}$ decays, perhaps $0.1 \%-1 \%$. Depending on what is the lightest supersymmetric partner, the signature will be $\mathrm{Z} \rightarrow \mathrm{h}+$ missing momentum, perhaps accompanied by one or two isolated hard $\gamma$ 's. Decays involving both the Higgs scalar $h$ and the pseudoscalar $\mathrm{A}$ are discussed, as well as ways to distinguish between them.
\end{abstract}

\section{Introduction}

Many particle physicists believe it is a real possibility that nature will become supersymmetric on the weak scale, with super partners having masses in the 100 $\mathrm{GeV}$ range. The motivations for this view and its phenomenological consequences have been widely studied [1,2]. In this note we point out that if nature indeed behaves this way, then there is a new possibility to produce and detect a Higgs boson. The relevant masses and rates are model dependent, but in a variety of models they can give a Higgs boson signal considerably exceeding that of the standard model.

In a supersymmetric theory the number of particles is doubled. In addition, weak interaction eigenstates and mass eigenstates do not coincide. Consequently, there is a considerable amount of new notation that has to be introduced to describe the

* Research supported in part by US Department of Energy.

${ }^{1}$ On leave of absence from the University of Michigan, Ann Arbor, MI 48109, USA.

${ }^{2}$ On leave of absence from Instituto de Estructura de la Materia, Serrano 119, 28006 Madrid, Spain. 
situation without oversimplification. Our notation will be as mnemonic as possible, but it is necessary to define a number of quantities in order to state the result precisely.

\section{Higgs bosons in the minimal supersymmetric model}

Two complex Higgs doublets are necessary in a supersymmetric theory [3-6]: $\mathrm{H}_{1}$ coupled to down-quarks and leptons, and $\mathrm{H}_{2}$ coupled to up-quarks. After the Higgs mechanism gives mass to the $\mathrm{W}^{ \pm}$and $\mathrm{Z}^{0}$ gauge bosons, five physical scalars remain - two charged Higgs bosons $\mathrm{H}^{ \pm}$, and three neutral Higgs bosons which we call $\mathrm{h}, \mathrm{H}, \mathrm{A}$. The first two are scalars, $\mathrm{h}$ the lighter and $\mathrm{H}$ the heavier, and the third is a pseudoscalar. There are two vacuum expectation values, $v_{1}$ and $v_{2}$, and we will only need their ratio, in the form of an angle $\beta$,

$$
\tan \beta=v_{2} / v_{1} \text {. }
$$

The diagonalization of the Higgs weak eigenstates into mass eigenstates involves another angle, $\alpha$, which can be written

$$
\sin 2 \alpha=-\sin 2 \beta\left(\frac{m_{\mathrm{H}}^{2}+m_{\mathrm{h}}^{2}}{m_{\mathrm{H}}^{2}-m_{\mathrm{h}}^{2}}\right) .
$$

Choosing a phase convention where $v_{1}$ and $v_{2}$ are real and positive, so $0 \leqslant \beta \leqslant \frac{1}{2} \pi$, we can choose $-\frac{1}{2} \pi \leqslant \alpha \leqslant 0$. Another way to relate these angles to the masses is [7]

$$
\cos (\beta-\alpha)=\operatorname{sign}\left(v_{1}-v_{2}\right)\left(\frac{m_{\mathrm{h}}^{2}\left(m_{\mathrm{Z}}^{2}-m_{\mathrm{h}}^{2}\right)}{\left(m_{\mathrm{H}}^{2}-m_{\mathrm{h}}^{2}\right)\left(m_{\mathrm{H}}^{2}+m_{\mathrm{h}}^{2}-m_{\mathrm{Z}}^{2}\right)}\right)^{1 / 2} .
$$

Although the masses are model dependent, normally $m_{\mathrm{H}}>m_{\mathrm{Z}}, m_{\mathrm{h}}<m_{\mathrm{Z}}$, with $m_{\mathrm{h}}$ typically being $10 \mathrm{GeV}$ to within a factor of two. The masses which appear in eq. (3) are tree-level masses, not including radiative corrections (which can be a large contribution to $m_{\mathrm{h}}$ ). In the minimal supersymmetric model, the mass of $\mathrm{A}$ is given by $m_{\mathrm{A}}^{2}=m_{\mathrm{h}}^{2}+m_{\mathrm{H}}^{2}-m_{\mathrm{Z}}^{2}$ and may be either heavier or lighter than the $\mathrm{Z}$.

There is also mixing of the neutralino states [8]. The weak interaction eigenstates are $\tilde{\mathbf{W}}_{3}, \tilde{\mathbf{B}}, \tilde{\mathbf{H}}_{1}, \tilde{\mathbf{H}}_{2}$. The mass matrix is complicated and normally must be diagonalized numerically. However, for a certain choice of parameters, the diagonalization may be carried out analytically to good approximation, resulting in the following approximate mass eigenstates $[9,10]$ :

$$
\begin{aligned}
& \tilde{\gamma}=\tilde{\mathrm{W}}_{3} \sin \theta_{\mathrm{W}}+\tilde{\mathrm{B}} \cos \theta_{\mathrm{W}}, \\
& \tilde{\mathrm{h}}=\tilde{\mathrm{H}}_{1} \sin \beta+\tilde{\mathrm{H}}_{2} \cos \beta,
\end{aligned}
$$


and

$$
\begin{aligned}
& \tilde{\mathbf{Z}}=\gamma_{5}\left[-\left(\tilde{\mathrm{W}}_{3} \cos \theta_{\mathrm{W}}-\tilde{\mathrm{B}} \sin \theta_{\mathrm{W}}\right) \sin \varphi+\left(\tilde{\mathrm{H}}_{1} \cos \beta-\tilde{\mathrm{H}}_{2} \sin \beta\right) \cos \varphi\right], \\
& \tilde{\mathrm{Z}}=\left(\tilde{\mathrm{W}}_{3} \cos \theta_{\mathrm{W}}-\tilde{\mathrm{B}} \sin \theta_{\mathrm{W}}\right) \cos \varphi+\left(\tilde{\mathrm{H}}_{1} \cos \beta-\tilde{\mathrm{H}}_{2} \sin \beta\right) \sin \varphi .
\end{aligned}
$$

These states are mass eigenstates in the limit where $m_{\tilde{\gamma}}, m_{\tilde{\mathrm{h}}} \ll m_{\mathrm{Z}}$. In addition

$$
\begin{aligned}
m_{\tilde{z}, \tilde{z}} & =\left(\mathrm{m}_{Z}^{2}+\frac{1}{4} m_{\tilde{\gamma}}^{2}\right)^{1 / 2} \pm \frac{1}{2} m_{\tilde{\gamma}}, \\
\tan 2 \varphi & =2 m_{Z} / m_{\tilde{\gamma}} .
\end{aligned}
$$

The notation has been chosen here to remind the reader of the various sizes of masses: $\tilde{\mathrm{h}}$ is light, $m_{\tilde{\mathrm{z}}}<m_{\mathrm{Z}}$ and $m_{\tilde{\mathrm{Z}}}>m_{\mathrm{Z}}$. However, the notation can be misleading, since neither $\tilde{\mathbf{z}}$ nor $\tilde{Z}$ is precisely the supersymmetric partner of the $Z$. Furthermore, $\tilde{h}$ is in fact the supersymmetric partner of the pseudoscalar Higgs boson, $\mathrm{A}$. The phenomenological consequences of a supersymmetric theory depend on what is the lightest supersymmetric particle (LSP). It can be either $\tilde{\gamma}, \tilde{\mathrm{h}}$, or $\tilde{\nu}$. For our purposes all that is affected is the signature for the Higgs; we will discuss the alternatives in context below.

We will generally guide our thinking by using numerical values that arise commonly in models. We will assume $v_{1} \approx v_{2}$, though $v_{1}=\frac{1}{2} v_{2}$ is certainly reasonable. We take $m_{\mathrm{h}} \ll m_{\mathrm{Z}}, m_{\mathrm{H}}$. The pseudoscalar Higgs mass $m_{\mathrm{A}}$ is unconstrained, depending on other aspects of the Higgs potential; it can be heavier or lighter than the $\mathrm{Z}$. We assume $m_{\tilde{\gamma}} \ll m_{\mathrm{Z}}$, though $\tilde{\gamma}$ could be heavier than $\tilde{h}$. Given these choices, one should keep in mind that one approximately has:

$$
\begin{gathered}
-\alpha \approx \beta \simeq \varphi \simeq \frac{1}{4} \pi \\
\cos ^{2}(\beta-\alpha) \simeq m_{\mathrm{h}}^{2} m_{\mathrm{Z}}^{2} / m_{\mathrm{H}}^{2}\left(m_{\mathrm{H}}^{2}-m_{\mathrm{Z}}^{2}\right) .
\end{gathered}
$$

From eq. (8), $\cos ^{2}(\beta-\alpha) \ll 1$ unless $m_{\mathrm{H}}^{2} \approx m_{\mathrm{Z}}^{2}$.

\section{Supersymmetric decays of Z's and z̃'s}

In this section we give the decays rates in terms of the quantities defined above. Since all are tree-level decays, the results follow simply from the Feynman rules, which are given in refs. $[9,10]$.

(a) $\tilde{z}$ decays. The largest $\tilde{z}$ decay is $\tilde{z} \rightarrow \nu \tilde{\nu}$, which will be the dominant decay whenever it is kinematically allowed. The rate, conveniently normalized to $\Gamma\left(\mathrm{Z}^{0} \rightarrow\right.$ $\nu \bar{\nu}) \approx 150 \mathrm{MeV}$ for one neutrino family, is:

$$
\frac{\Gamma(\tilde{\mathrm{z}} \rightarrow \nu \tilde{\nu})}{\Gamma(\mathrm{Z} \rightarrow \nu \bar{\nu})}=\frac{3 m_{\tilde{\mathrm{z}}}}{4 m_{\mathrm{Z}}} \sin ^{2} \varphi\left(1-m_{\tilde{\nu}}^{2} / m_{\tilde{\mathrm{z}}}^{2}\right)^{2} .
$$


Other possible two-body decays are:

$$
\begin{aligned}
& \frac{\Gamma(\tilde{\mathrm{z}} \rightarrow \mathrm{A} \tilde{\mathrm{h}})}{\Gamma(\tilde{\mathrm{z}} \rightarrow \nu \tilde{\nu})}=\frac{2 \cos ^{2} 2 \beta\left(1-m_{\mathrm{A}}^{2} / m_{\tilde{z}}^{2}\right)^{2}}{\left(1-m_{\tilde{\nu}}^{2} / m_{\tilde{\mathrm{z}}}^{2}\right)^{2}}, \\
& \frac{\Gamma(\tilde{\mathrm{z}} \rightarrow \mathrm{h} \tilde{\mathrm{h}})}{\Gamma(\tilde{\mathrm{z}} \rightarrow \nu \tilde{\nu})}=\frac{2 \cos ^{2}(\beta-\alpha)\left(1-m_{\mathrm{h}}^{2} / m_{\tilde{\mathrm{z}}}^{2}\right)^{2}}{\left(1-m_{\tilde{\nu}}^{2} / m_{\tilde{\mathrm{z}}}^{2}\right)^{2}} .
\end{aligned}
$$

If it is kinematically allowed, the most important three-body decay is:

$$
\Gamma(\tilde{\mathrm{z}} \rightarrow \mathrm{q} \overline{\mathrm{q}} \tilde{\mathrm{g}})=\frac{\alpha_{\mathrm{s}} g_{2}^{2} m_{\tilde{\mathrm{z}}}^{5} \sin ^{2} \varphi}{48 \pi^{2} \cos ^{2} \theta_{\mathrm{w}} m_{\tilde{\mathrm{q}}}^{4}}\left[\left(T_{3 \mathrm{q}}-e_{\mathrm{q}} \sin ^{2} \theta_{\mathrm{w}}\right)^{2}+e_{\mathrm{q}}^{2} \sin ^{4} \theta_{\mathrm{w}}\right] .
$$

If we sum eq. (12) over $u, d, c, s, b$, we get

$$
\frac{\sum_{\mathrm{q}} \Gamma(\tilde{\mathrm{z}} \rightarrow \mathrm{q} \overline{\mathrm{q}} \tilde{\mathrm{g}})}{\Gamma(\tilde{\mathrm{z}} \rightarrow \nu \tilde{\nu})} \simeq \frac{2.3 \alpha_{\mathrm{s}}}{\pi}\left(\frac{m_{\tilde{\mathrm{z}}}}{m_{\tilde{\mathrm{q}}}}\right)^{4} \frac{1}{\left(1-m_{\nu}^{2} / m_{\tilde{\mathrm{z}}}^{2}\right)^{2}} .
$$

Comparing these, it is clear that $\tilde{\mathrm{z}} \rightarrow \mathrm{h} \tilde{\mathrm{h}}$ or $\tilde{\mathrm{z}} \rightarrow \mathrm{A} \tilde{\mathrm{h}}$ can easily dominate if $\cos ^{2} 2 \beta$ or $\cos ^{2}(\beta-\alpha)$ are not too small. Although these factors vanish in the limit given by eq. (7) deviations from this limit will lead to non-negligible results.

Finally, if the gluino is too heavy, we should consider $\tilde{z} \rightarrow q \bar{q} \tilde{\chi}$, where $\tilde{\chi}$ is either a chargino or neutralino lighter than the ż. However, unless the two-body decays are forbidden or very suppressed, these decay rates (which are proportional to the weak coupling constant squared, $g_{2}^{2}$ ) can be neglected.

(b) $Z$ decays. In models with two Higgs doublets, the $Z^{0}$ can decay into two neutral Higgs bosons. Bose symmetry forbids these two neutral Higgs bosons to be identical. Thus, in the minimal supersymmetric model, the only possible decay is $\mathrm{Z}^{0} \rightarrow \mathrm{hA}$. (If the Higgs sector conserves $C P$, then the two neutral bosons emitted in the Z-decay must have opposite $C P$ quantum numbers.) The decay rate into Higgs boson is then:

$$
\frac{\Gamma(\mathrm{Z} \rightarrow \mathrm{hA})}{\Gamma(\mathrm{Z} \rightarrow \nu \bar{\nu})}=\frac{1}{2} \cos ^{2}(\beta-\alpha) \lambda^{3 / 2}\left(m_{\mathrm{Z}}^{2}, m_{\mathrm{h}}^{2}, m_{\mathrm{A}}^{2}\right) / m_{\mathrm{Z}}^{6}
$$

where $\lambda(a, b, c) \equiv(a+b-c)^{2}-4 a b$. The supersymmetric analogue of eq. (14) is:

$$
\frac{\Gamma(\mathrm{Z} \rightarrow \tilde{\mathrm{h}} \tilde{\mathrm{h}})}{\Gamma(\mathrm{Z} \rightarrow \nu \bar{\nu})}=\cos ^{2} 2 \beta
$$


Thus, $\tilde{h} \tilde{h}$ can contribute at most as much as one extra neutrino. Since $\beta \approx 45^{\circ}$ in many models, this may be a rather small rate; but as an example, for $\tan \beta=2$, $\cos ^{2} 2 \beta=0.36$ giving about $\frac{1}{3}$ of a neutrino family.

We now turn to supersymmetric decays of the $Z$ involving the $\tilde{z}$. The only decay which is likely to be kinematically accessible is $Z \rightarrow \tilde{z} \tilde{h}$. Neglecting $m_{\tilde{h}}$, we find:

$$
\frac{\Gamma(\mathrm{Z} \rightarrow \tilde{\mathrm{z}} \tilde{\mathrm{h}})}{\Gamma(\mathrm{Z} \rightarrow \nu \bar{\nu})}=2 \cos ^{2} \varphi \sin ^{2} 2 \beta\left(1-\frac{m_{\tilde{\mathrm{z}}}^{2}}{m_{\mathrm{Z}}^{2}}\right)^{2}\left(1+\frac{m_{\tilde{\mathrm{z}}}^{2}}{2 m_{\mathrm{Z}}^{2}}\right) .
$$

If $m_{\tilde{\gamma}} \ll m_{\mathrm{Z}}$, and $\varphi \approx \beta \approx 45^{\circ}$, then

$$
\frac{\Gamma(\mathrm{Z} \rightarrow \tilde{\mathrm{z}} \tilde{\mathrm{h}})}{\Gamma(\mathrm{Z} \rightarrow \nu \bar{\nu})} \approx \frac{3 m_{\tilde{y}}^{2}}{2 m_{\mathrm{Z}}^{2}} .
$$

The $\tilde{\mathbf{z}}$ will then decay as discussed above. It is convenient to express the full decay chain in the following form:

$$
\begin{aligned}
\frac{\Gamma(\mathrm{Z} \rightarrow \tilde{\mathrm{h}} \tilde{\mathrm{h}} \mathrm{X})}{\Gamma(\mathrm{Z} \rightarrow \nu \bar{\nu})}= & \frac{\Gamma(\mathrm{Z} \rightarrow \tilde{\mathrm{z}} \tilde{\mathrm{h}})}{\Gamma(\mathrm{Z} \rightarrow \nu \bar{\nu})} \\
& \times \frac{\Gamma(\tilde{\mathrm{z}} \rightarrow \mathrm{X} \tilde{\mathrm{h}})}{\Gamma(\tilde{\mathrm{z}} \rightarrow \nu \tilde{\nu})+\Gamma(\tilde{\mathrm{z}} \rightarrow \mathrm{q} \overline{\mathrm{q}} \tilde{\mathrm{g}})+\Gamma(\tilde{\mathrm{z}} \rightarrow \mathrm{h} \tilde{\mathrm{h}})+\Gamma(\tilde{\mathrm{z}} \rightarrow \mathrm{A} \tilde{\mathrm{h}})} .
\end{aligned}
$$

where $\mathrm{X}=\mathrm{h}$ or A. Which terms occur in the denominator of the second factor is model dependent. If $\tilde{\mathrm{z}} \rightarrow \nu \tilde{\nu}$ is kinematically allowed, it dominates and the other two terms can be neglected. Then we get (neglecting $m_{\tilde{\mathrm{h}}}$ ):

$$
\begin{aligned}
\frac{\Gamma(\mathrm{Z} \rightarrow \tilde{\mathrm{h}} \tilde{\mathrm{h} X})}{\Gamma(\mathrm{Z} \rightarrow \nu \bar{\nu})} \approx & 4 F \cos ^{2} \varphi \sin ^{2} 2 \beta \\
& \times\left(1-\frac{m_{\tilde{\mathrm{z}}}^{2}}{m_{\mathrm{Z}}^{2}}\right)^{2}\left(1+\frac{m_{\tilde{\tilde{z}}}^{2}}{2 m_{\mathrm{Z}}^{2}}\right)\left(1-\frac{m_{\mathrm{X}}^{2}}{m_{\tilde{\mathrm{z}}}^{2}}\right)^{2}\left(1-\frac{m_{\tilde{\nu}}^{2}}{m_{\tilde{\mathrm{z}}}^{2}}\right)^{-2},
\end{aligned}
$$

where

$$
F \equiv \begin{cases}\cos ^{2}(\beta-\alpha), & \mathrm{X}=\mathrm{h} \\ \cos ^{2} 2 \beta, & \mathrm{X}=\mathrm{A} .\end{cases}
$$

If $\tilde{\mathbf{z}} \rightarrow \nu \tilde{\nu}$ is forbidden, then $\tilde{\mathbf{z}} \rightarrow \mathrm{h} \tilde{h}$ or $A \tilde{h}$ has to be compared to $\tilde{\mathbf{z}} \rightarrow \mathrm{q} \overline{\mathrm{q}} \tilde{\mathrm{g}}$, assuming the gluino is light enough so that the latter decay occurs. Given the current mass limits on gluinos $\left(M_{\tilde{\mathrm{g}}} \simeq 60 \mathrm{GeV}\right)$ obtained from the analysis of UA1 missing energy events [11], we believe it is reasonable to assume that $\tilde{z} \rightarrow h \tilde{h}$, or $A \tilde{h}$ 
dominate $^{\star}$, in which case:

$$
\frac{\Gamma(\mathrm{Z} \rightarrow \tilde{\mathrm{h}} \tilde{\mathrm{h} X})}{\Gamma(\mathrm{Z} \rightarrow \nu \bar{\nu})} \simeq \frac{\Gamma(\mathrm{Z} \rightarrow \tilde{\mathrm{z}} \tilde{\mathrm{h}})}{2 \Gamma(\mathrm{Z} \rightarrow \nu \bar{\nu})} \simeq \cos ^{2} \varphi \sin ^{2} 2 \beta\left(1-\frac{m_{\tilde{\mathrm{z}}}^{2}}{m_{\mathrm{Z}}^{2}}\right)^{2}\left(1+\frac{m_{\tilde{\mathrm{z}}}^{2}}{2 m_{\mathrm{Z}}^{2}}\right)
$$

where the branching ratios are about equal to $X=h, A$. Note that eq. (20) is larger than eq. (19a) by a factor of $F$, defined in eq. (19b). Using eq. (8), this implies that $\mathrm{BR}(\mathrm{Z} \rightarrow \tilde{\mathrm{h}} \tilde{\mathrm{h}} \mathrm{h})$ is enhanced in this case by typically an order of magnitude, with no falloff at small $m_{\mathrm{h}}$ (as long as the condition mentioned in the footnote on this page is satisfied).

The result from eq. (19) is shown in figs. 1 to 4 for a range of masses for $h$ and $A$, using the two models described in the appendix (which are variations of the model given in ref. [9]). Note that the decay $Z^{0} \rightarrow \tilde{h} \tilde{h} h$ dominates the usual $h$ decay of $Z^{0}$ for $m_{\mathrm{h}} \geqslant 0.1 m_{\mathrm{Z}}$, i.e. for essentially the entire region of interest at SLC/LEP, and is about two orders of magnitude larger in rate for much of the range. The falloff of the curves in figs. 1 and 3 at small $m_{\mathrm{h}}$ is due to the factor of $m_{\mathrm{h}}^{2}$ in eq. (3), and the falloff at large $m_{\mathrm{h}}$ is due to the fact that $\sin 2 \beta \rightarrow 0$ as $m_{\mathrm{h}} \rightarrow m_{\mathrm{Z}}$. If $\tilde{\mathrm{z}} \rightarrow \nu \tilde{\nu}$ is kinematically forbidden, the result is perhaps an order of magnitude larger and does not fall off at small $m_{\mathrm{h}}$, as shown by eq. (20). Similar remarks can be made for the decay $Z^{0} \rightarrow \tilde{h} \tilde{h} A$. In this case, due to the absence of a tree-level $Z Z A$ vertex, this may be the only way to produce the A at SLC/LEP at a detectable rate!

\section{Signatures and comments}

The signature(s) for finding the Higgs depend on what is the LSP, so we describe them one at a time. First we consider the decays of $h$ and A. To establish that $h$ is a Higgs boson, it is crucial to measure the decay to pairs of different fermion flavors to verify that $\mathrm{h}$ couples proportionally to mass. Presumably the $\tau \bar{\tau}$ mode will be the second flavor seen, since separating $c \bar{c}$ and $b \bar{b}$ will be difficult. The branching ratios depend on color, on mass, and on the Higgs sector:

$$
\begin{gathered}
\Gamma(\mathrm{h} \rightarrow \tau \bar{\tau})=\frac{g_{2}^{2} \sin ^{2} \alpha}{32 \pi \cos ^{2} \beta} \frac{m_{\tau}^{2}}{m_{\mathrm{w}}^{2}}\left(1-\frac{4 m_{\tau}^{2}}{m_{\mathrm{h}}^{2}}\right)^{3 / 2} m_{\mathrm{h}}, \\
\Gamma(\mathrm{h} \rightarrow \mathrm{c} \overline{\mathrm{c}})=\frac{3 g_{2}^{2} \cos ^{2} \alpha}{32 \pi \sin ^{2} \beta} \frac{m_{\mathrm{c}}^{2}}{m_{\mathrm{W}}^{2}}\left(1-\frac{4 m_{\mathrm{c}}^{2}}{m_{\mathrm{h}}^{2}}\right)^{3 / 2} m_{\mathrm{h}},
\end{gathered}
$$

\footnotetext{
ॠThis conclusion is valid only if $\cos ^{2}(\beta-\alpha)$ or $\cos ^{2} 2 \beta$ are not too small. Otherwise, as mentioned earlier, the decay $\tilde{z} \rightarrow q \bar{q} \tilde{\chi}$ can become important, thereby making eqs. (18) and (20) invalid. In this discussion, we implicitly assume that the latter decay can be neglected.
} 


$$
\begin{aligned}
& \Gamma(\mathrm{h} \rightarrow \mathrm{b} \overline{\mathrm{b}})=\frac{3 g_{2}^{2} \sin ^{2} \alpha}{32 \pi \cos ^{2} \beta} \frac{m_{\mathrm{b}}^{2}}{m_{\mathrm{W}}^{2}}\left(1-\frac{4 m_{\mathrm{b}}^{2}}{m_{\mathrm{h}}^{2}}\right)^{3 / 2} m_{\mathrm{h}}, \\
& \Gamma(\mathrm{A} \rightarrow \tau \bar{\tau})=\frac{g_{2}^{2} \tan ^{2} \beta}{32 \pi} \frac{m_{\tau}^{2}}{m_{\mathrm{W}}^{2}}\left(1-\frac{4 m_{\tau}^{2}}{m_{\mathrm{A}}^{2}}\right)^{1 / 2} m_{\mathrm{A}} \\
& \Gamma(\mathrm{A} \rightarrow \mathrm{c} \overline{\mathrm{c}})=\frac{3 g_{2}^{2} \cot ^{2} \beta}{32 \pi} \frac{m_{\mathrm{c}}^{2}}{m_{\mathrm{W}}^{2}}\left(1-\frac{4 m_{\mathrm{c}}^{2}}{m_{\mathrm{A}}^{2}}\right)^{1 / 2} m_{\mathrm{A}} \\
& \Gamma(\mathrm{A} \rightarrow \mathrm{b} \overline{\mathrm{b}})=\frac{3 g_{2}^{2} \tan ^{2} \beta}{32 \pi} \frac{m_{\mathrm{b}}^{2}}{m_{\mathrm{W}}^{2}}\left(1-\frac{4 m_{\mathrm{b}}^{2}}{m_{\mathrm{A}}^{2}}\right)^{1 / 2} m_{\mathrm{A}}
\end{aligned}
$$

If $-\alpha \simeq \beta \simeq \frac{1}{4} \pi$, then these ratios will be the usual standard model ones, but that need not be the case. The mode c $\bar{c}$ can be suppressed or enhanced relative to $\tau \bar{\tau}$ and $\mathrm{b} \overrightarrow{\mathrm{b}}$ (e.g. for $v_{2} \gg v_{1}$, then $\cos ^{2} \beta \ll 1$ implying suppressed $\mathrm{c} \overline{\mathrm{c}}$ production and enhanced $\tau \bar{\tau}$ and $b \bar{b}$ production).

Note that there is a clean kinematical way to distinguish between a pseudoscalar or scalar if a single Higgs is found, since

$$
\Gamma(\mathrm{h} \rightarrow \tau \bar{\tau}) / \Gamma(\mathrm{h} \rightarrow \mathrm{b} \overline{\mathrm{b}})=\frac{m_{\tau}^{2}}{3 m_{\mathrm{b}}^{2}}\left(\frac{1-4 m_{\tau}^{2} / m_{\mathrm{h}}^{2}}{1-4 m_{\mathrm{b}}^{2} / m_{\mathrm{h}}^{2}}\right)^{3 / 2}
$$

while

$$
\Gamma(\mathrm{A} \rightarrow \tau \bar{\tau}) / \Gamma(\mathrm{A} \rightarrow \mathrm{b} \overline{\mathrm{b}})=\frac{m_{\tau}^{2}}{3 m_{\mathrm{b}}^{2}}\left(\frac{1-4 m_{\tau}^{2} / m_{\mathrm{A}}^{2}}{1-4 m_{\mathrm{b}}^{2} / m_{\mathrm{A}}^{2}}\right)^{1 / 2}
$$

These ratios differ by a factor of 1.65 for $m_{\mathrm{A}}$ or $m_{\mathrm{h}}=14 \mathrm{GeV}$, a typical value from models. Thus comparison of the leptonic and hadronic "down" modes will unambiguously tell us the parity of the Higgs ${ }^{\star}$, with important implications for interpretations. In practice, both $h$ and $A$ could occur in a superysmmetric world, so care should be taken to allow for a double peaking. Then from the various branching ratios and masses one can untangle all of the quantum numbers.

The signatures will vary depending on what is the LSP. If $\tilde{\mathrm{h}}$ or $\tilde{\nu}$ is the LSP, $\tilde{\mathrm{h}}$ will either escape or decay to $\tilde{\nu} \nu$ which escape, so the signature is $Z \rightarrow h+$ missing

* If $v_{2} \gg v_{1}$, this comparison is cleanest since all hadronic modes will be into "down-type" quarks. 
momentum, with $\mathrm{h}$ decaying as above. If the photino is the $\mathrm{LSP}^{\star}$, then $\tilde{\mathrm{h}} \rightarrow \gamma \tilde{\gamma}$, with $\tilde{\gamma}$ escaping. The $\tilde{\mathrm{h}} \rightarrow \gamma \tilde{\gamma}$ decay is a flat two-body decay, so the full event can have $\mathrm{Z} \rightarrow \mathrm{h}+$ missing momentum $+(0,1$, or 2$)$ photons. Since good models exist with any of the above as LSP, all these signatures should be examined experimentally. If the photino is not the LSP, there are strong constraints from the non-observation of the process $\mathrm{e}^{+} \mathrm{e}^{-} \rightarrow \tilde{\gamma} \tilde{\gamma}$ (via $\tilde{\mathrm{e}}$ exchange) followed by $\tilde{\gamma} \rightarrow \gamma \tilde{\mathrm{h}}$. Such events, which would be observed as $\mathrm{e}^{+} \mathrm{e}^{-} \rightarrow \gamma \gamma+$ missing energy, have not been seen [12]. If one assumes that the photino decays radiatively, it follows that $m_{\tilde{e}} \geq 100 \mathrm{GeV}$. Alternatively, if the $\tilde{\nu}$ were the LSP, the photino would decay via $\tilde{\gamma} \rightarrow \tilde{\nu} \nu$ and the above constraints would not be relevant. We have checked that all the cases considered in this paper are not in conflict with the experimental results of ref. [12].

Another amusing possibility arises from noting that if $m_{\mathrm{h}}>2 m_{\tilde{v}}$, then:

$$
\Gamma(\mathrm{h} \rightarrow \tilde{\nu} \overline{\tilde{\nu}})=\frac{g_{2}^{2} m_{\mathrm{Z}}^{2}}{64 \pi m_{\mathrm{h}} \cos ^{2} \theta_{\mathrm{W}}} \sin ^{2}(\alpha+\beta)\left(1-4 m_{\tilde{\nu}}^{2} / m_{\mathrm{h}}^{2}\right)^{1 / 2},
$$

while

$$
\Gamma(\mathrm{A} \rightarrow \tilde{\nu} \overline{\tilde{\nu}})=0 .
$$

Then one can have (see eq. (14)) a large branching ratio for $Z \rightarrow h A$, followed by $h$ decaying as in eq. (29) and thus disappearing, and A decaying normally, giving a similar signature to our main one from a different source. For our canonical values of $-\alpha \approx \beta$ the rate for $h \rightarrow \tilde{\nu} \tilde{\tilde{\nu}}$ is suppressed, but in general it may not be. All the decays we have been discussing have a one-sided or monojet signature, perhaps with hard isolated $\gamma$ 's.

Finally, we briefly mention another possible way in which supersymmetric particle decays can result in a Higgs boson in the final state. The idea is to find processes which produce the $\tilde{z}$ (either real or virtual) with high probability, which then can decay via $\tilde{z} \rightarrow h \tilde{h}$. We have already presented one such case, e.g. $Z^{0} \rightarrow \tilde{z} \tilde{h}$ $\rightarrow \tilde{h} \tilde{h} h$. Here is another example. Consider a model where $\tilde{h}$ is the LSP and the scalar electron ( $\tilde{e})$ is the second lightest supersymmetric particle. In particular, we demand that $m_{\tilde{\mathrm{e}}}<m_{\tilde{\gamma}}, m_{\tilde{\mathrm{z}}}$. The decay $\tilde{\mathrm{e}}^{ \pm} \rightarrow \mathrm{e}^{ \pm} \tilde{\mathrm{h}}$ is highly suppressed due to the suppression factor $m_{\mathrm{e}} / m_{\mathrm{W}}$ in the eẽ $\tilde{\mathrm{h}}$ coupling. The dominant decay mode of $\tilde{\mathrm{e}}^{ \pm}$

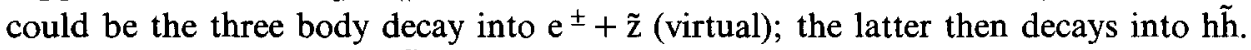
Not only does $\tilde{\mathrm{e}}^{ \pm} \rightarrow \mathrm{e}^{ \pm} \mathrm{h} \tilde{\mathrm{h}}$ provide a new mechanism for producing the Higgs boson; it also suggests a possible loophole in previously obtained experimental bounds for the scalar-electron mass [13]. Other examples can be constructed along similar lines.

* This case does not apply to the approximation (4) in which necessarily $m_{\dot{\gamma}}>2 m_{\overline{\mathrm{h}}}$. When the LSP is a neutralino it will in general have a nonvanishing component along $\tilde{\gamma}$, but we will not consider the general situation further in this paper. 


\section{Concluding remarks}

We have pointed out a way in which Higgs bosons would appear experimentally if there is low-energy supersymmetry in nature. The branching ratio for $\mathrm{Z} \rightarrow$ Higgs could be as much as two orders of magnitude larger than the standard model one, and the signatures are distinctive. It should also be kept in mind that if $\tilde{h}$ is the LSP, then it can happen that $\tilde{\gamma} \rightarrow \gamma \tilde{h}$; so supersymmetry can give rise to isolated photons plus missing momentum at $\mathrm{e}^{+} \mathrm{e}^{-}$or at hadron colliders.

\section{Appendix}

In this appendix we will briefly describe a phenomenological low-energy supergravity model which can produce a sizeable decay rate for $Z^{0} \rightarrow \tilde{h} \tilde{h} h$ and $Z^{0} \rightarrow \tilde{h} \tilde{h} A$. The model is a generalization of the one presented in ref. [14] where a general supergravity lagrangian is used, which implies that not only the $\mathrm{SU}(3), \mathrm{SU}(2)$ and U(1) gaugino Majorana masses, but also the gauge coupling constants are not universal at the grand unification scale.

In ref. [14] we showed that no supersymmetric Higgs mixing parameter $\mu$ was needed to radiatively break $\mathrm{SU}(2) \times \mathrm{U}(1)$ with a light top quark, $m_{\mathrm{t}}<55 \mathrm{GeV}$, provided that we do not artificially impose the condition of universality of gaugino Majorana masses at $M_{\mathrm{X}}$. Further, the minimal supersymmetric model predicts $\sin ^{2} \theta_{\mathrm{W}}\left(m_{\mathrm{W}}\right)=0.236$ while the most recent measurements of $m_{\mathrm{W}}$ by the UA1 Collaboration [15] give $\sin ^{2} \theta_{\mathrm{W}}\left(m_{\mathrm{W}}\right)=\left(38.64 / m_{\mathrm{W}}\right)^{2}=0.216$ in good agreement with the world average $\sin ^{2} \theta_{\mathrm{w}} \simeq 0.215$. It was observed in ref. [16] that the correct value of $\sin ^{2} \theta_{\mathrm{w}}$ could indeed be obtained by relaxing the universality of gauge coupling constants at $M_{\mathrm{X}}$.

The present model is characterized by a non-trivial function $f_{\alpha \beta}$, such that $\left\langle f_{\alpha \beta}\right\rangle \neq \delta_{\alpha \beta}$, implying that the gauge coupling constants need to be rescaled and are not equal at $M_{\mathrm{X}}$. Furthermore, $\left\langle\partial f_{\alpha \beta} / \partial \phi^{a}\right\rangle \neq 0$ leading to gaugino Majorana masses given by ref. [17] (assuming canonical kinetic terms in the chiral sector)

$$
m_{\alpha \beta}=\frac{1}{2} \mathrm{e}^{G / 2} G^{a} \frac{\partial f_{\alpha \beta}^{*}}{\partial \phi_{a}}
$$

where $G$ is the Kähler potential and $\phi^{a}$ are all scalar fields of the theory. Depending on the particular choice of $f_{\alpha \beta}, m_{\alpha \beta}\left(M_{\mathrm{X}}\right)$ may be different for SU(3), SU(2) or U(1) indices. Here we will assume that $\mathrm{SU}(5)$ is the grand unification group and use two particular choices [16] of $f_{\alpha \beta}$ depending on whether $\mathrm{SU}(5)$ is broken with the 24 Higgs $\Sigma^{\alpha}$,

$$
f_{\alpha \beta}^{(24)}=A\left(\phi^{a}\right) \delta_{\alpha \beta}+B\left(\phi^{a}\right) d_{\alpha \beta \gamma} \Sigma^{\gamma},
$$


or with the 75 Higgs $\Sigma_{c d}^{[a b]}$

$$
f_{\alpha \beta}^{(75)}=A\left(\phi^{a}\right) \delta_{\alpha \beta}+B\left(\phi^{a}\right) \lambda_{[a}^{\alpha c} \lambda_{b]}^{\beta d} \Sigma_{c d}^{[a b]},
$$

where $A$ and $B$ are invariant functions of scalar fields.

Using eqs. (A.1)-(A.3), all GUT predictions can be written as functions of a real parameter $x$. At one-loop one gets

$$
\begin{aligned}
\log \frac{M_{\mathrm{X}}}{Q} & =\frac{2 \pi}{D}\left[\alpha_{\mathrm{em}}^{-1}(Q)-B \alpha_{3}^{-1}(Q)\right], \\
\alpha_{3}^{-1}\left(M_{\mathrm{X}}\right) & =\alpha_{3}^{-1}(Q)+\frac{b_{3}}{D}\left[\alpha_{\mathrm{em}}^{-1}(Q)-B \alpha_{3}^{-1}(Q)\right], \\
\sin ^{2} \theta_{\mathrm{w}}(Q) & =C \alpha_{\mathrm{em}}(Q) \alpha_{3}^{-1}(Q)+\frac{1}{D}\left[1-B \alpha_{\mathrm{em}}(Q) \alpha_{3}^{-1}(Q)\right]\left[C b_{3}-b_{2}\right], \\
\alpha_{1}\left(M_{\mathrm{X}}\right) & =\alpha_{3}\left(M_{\mathrm{X}}\right) / x, \\
\alpha_{2}\left(M_{\mathrm{X}}\right) & =\alpha_{3}\left(M_{\mathrm{X}}\right) / C,
\end{aligned}
$$

where $b_{i}(i=1,2,3)$ are the renormalization group coefficients,

$$
D(x)=b_{3} B(x)-b_{2}-\frac{5}{3} b_{1}
$$

and $B(x), C(x)$ depend on the mechanism chosen to break SU(5). One finds

$$
\begin{aligned}
& B^{(24)}(x)=\frac{2}{3}(5 x-1), \\
& B^{(75)}(x)=\frac{4}{3}(1+x),
\end{aligned}
$$

and

$$
\begin{aligned}
& C^{(24)}(x)=\frac{1}{3}(5 x-2), \\
& C^{(75)}(x)=\frac{1}{3}(4-x) .
\end{aligned}
$$

For the minimal model $\left(b_{3}=3, b_{2}=-1, b_{1}=-6.6\right)$ and using as boundary conditions $\alpha_{\mathrm{em}}\left(m_{\mathrm{W}}\right)=1 / 127.54$ and $\alpha_{3}\left(m_{\mathrm{W}}\right)=0.12$, we obtain from eqs. (A.4)-(A.7), and fixing $\sin ^{2} \theta_{\mathrm{w}}\left(m_{\mathrm{W}}\right)=0.215$, the following predictions:

(i) For the 24-mechanism, eq. (A.2), $x=0.921$ which corresponds to

$$
\begin{gathered}
M_{\mathrm{X}}=1.55 \times 10^{17} \mathrm{GeV} \\
\alpha_{3}^{-1}\left(M_{\mathrm{X}}\right)=25.12, \quad \alpha_{2}^{-1}\left(M_{\mathrm{X}}\right)=21.82, \quad \alpha_{1}^{-1}\left(M_{\mathrm{X}}\right)=23.14 .
\end{gathered}
$$


(ii) For the 75-mechanism, eq. (A.3), $x=1.140$ which corresponds to

$$
\begin{gathered}
M_{\mathrm{X}}=4.90 \times 10^{15} \mathrm{GeV} \\
\alpha_{3}^{-1}\left(M_{\mathrm{X}}\right)=23.47, \quad \alpha_{2}^{-1}\left(M_{\mathrm{X}}\right)=22.38, \quad \alpha_{1}^{-1}\left(M_{\mathrm{X}}\right)=26.76 .
\end{gathered}
$$

The ratio of gaugino Majorana masses at $M_{\mathrm{X}}$ can be given, from eqs. (A.1)-(A.3) as a function of a real variable $\lambda$ :

$$
\frac{m_{3}\left(M_{\mathrm{X}}\right)}{1}=\frac{m_{2}\left(M_{\mathrm{X}}\right)}{\lambda / C(x)}=\frac{m_{1}\left(M_{\mathrm{X}}\right)}{\rho / x}
$$

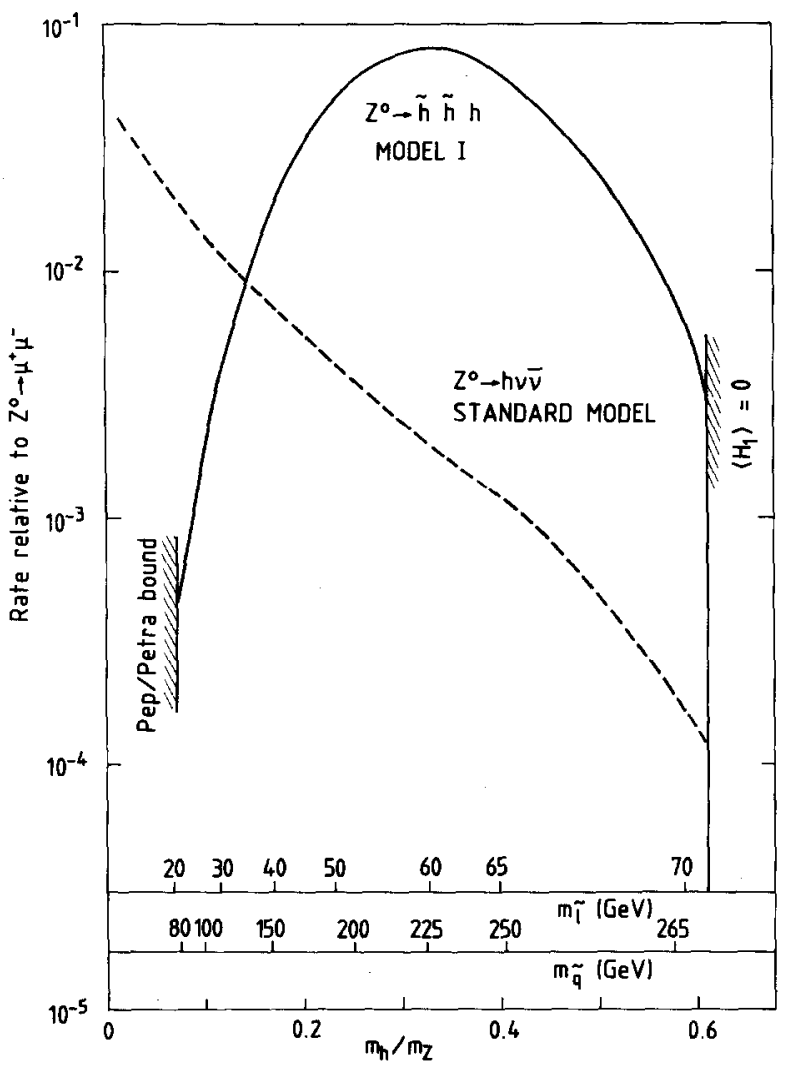

Fig. 1. Decay rate $\Gamma\left(\mathrm{Z}^{0} \rightarrow \tilde{\mathrm{h}} \mathrm{h} h\right) / \Gamma\left(\mathrm{Z}^{0} \rightarrow \mu^{+} \mu^{-}\right)$as a function of $m_{\mathrm{h}} / m_{\mathrm{Z}}$ for model I described in the appendix (SU(5) broken with the 24 Higgs). The chosen supergravity parameters are: $A=3, \lambda=0.4$, $\xi=1.8$ and $\mu=2 \mathrm{GeV}$. Typical values of slepton and squark masses are indicated on the lower horizontal axis. The shadowed region to the right of the vertical line $\left\langle\mathrm{H}_{1}\right\rangle=0$ corresponds to values $|\sin 2 \beta|>1$. For comparison, the decay rate for the standard model process $\Sigma \Gamma\left(\mathrm{Z}^{0} \rightarrow \mathrm{h} \nu \bar{\nu}\right) / \Gamma\left(\mathrm{Z}^{0} \rightarrow \mu^{+} \mu^{-}\right)$, summed over the three generations of neutrinos, is included. 
where

$$
\begin{aligned}
& \rho^{(24)}=\frac{1}{5}(2+3 \lambda), \\
& \rho^{(75)}=4-3 \lambda .
\end{aligned}
$$

We observe that the ratio given by eq. (A.10) is equal to one for $x=\lambda=1$, which provides the minimal gauge coupling. Hereafter we will consider $x$ fixed by the boundary conditions (eq. (A.8) or (A.9)) while $\lambda$ is a free parameter of the theory.

We will study two supergravity models: (i) Model I, with boundary conditions given by eqs. (A.8) and (A.11), corresponding to the breaking of SU(5) with the 24 Higgs, eq. (A.2). (ii) Model II, with boundary conditions given by eqs. (A.9) and (A.12), which assumes that $\mathrm{SU}(5)$ is broken with the 75 Higgs, eq. (A.3). For both models we will assume, as in ref. [14] that $\mu \ll m_{\mathrm{W}}$ (i.e. $\mu=0$ at tree level, but $\mu \neq 0$ generated by 1-loop radiative corrections in the superheavy sector), so that it cannot trigger the radiative breaking of $\mathrm{SU}(2) \times \mathrm{U}(1)$ if $\lambda=1$ and $m_{\mathrm{t}}<55 \mathrm{GeV}$. The

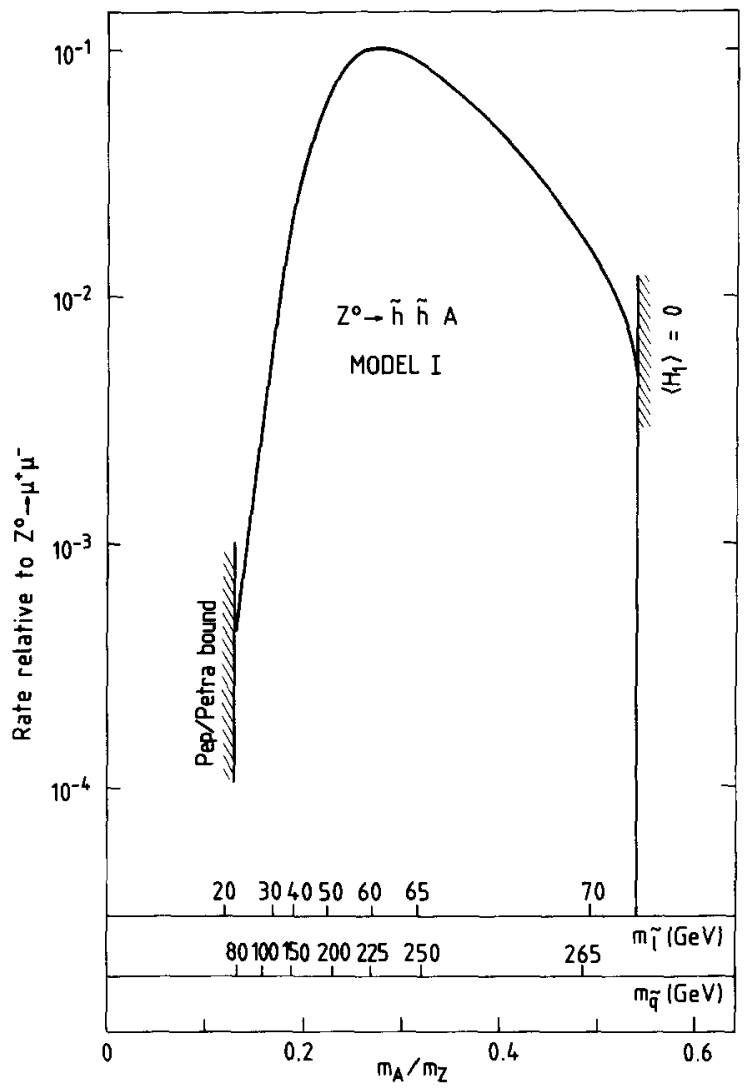

Fig. 2. Decay rate $\Gamma\left(\mathrm{Z}^{0} \rightarrow \tilde{\mathrm{h}} \tilde{\mathrm{h}} \mathrm{A}\right) / \Gamma\left(\mathrm{Z}^{0} \rightarrow \mu^{+} \mu^{-}\right)$as a function of $m_{\mathrm{A}} / m_{\mathrm{Z}}$ for model I. The supergravity parameters are as in fig. 1 . 


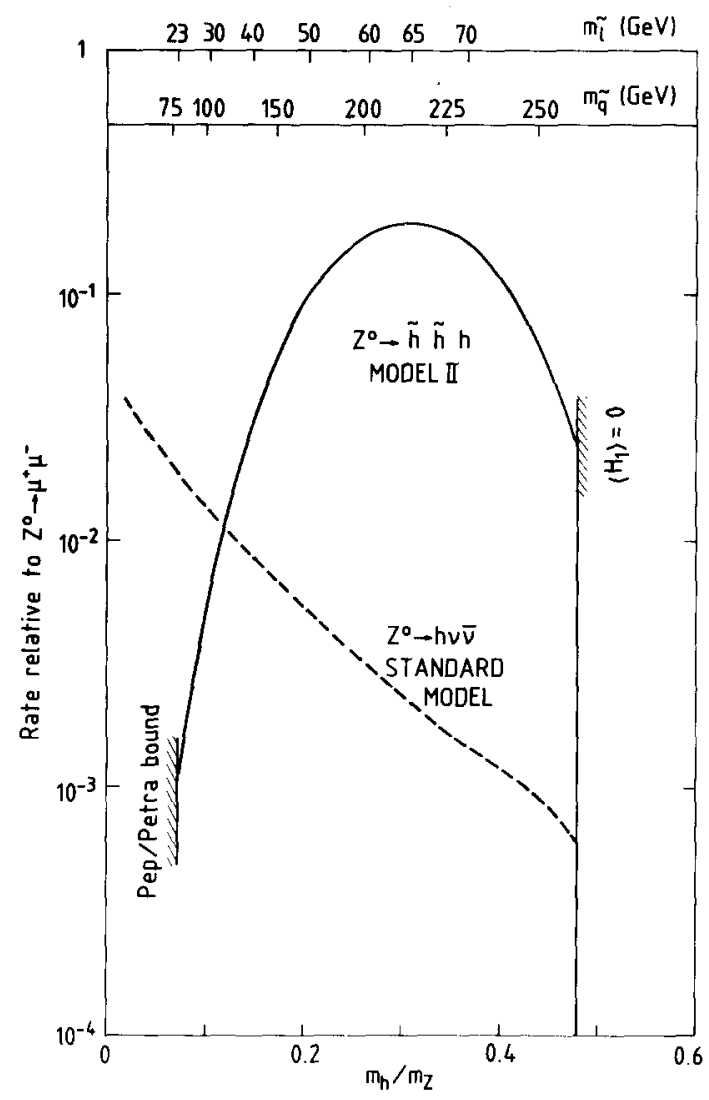

Fig. 3. Decay rate $\Gamma\left(\mathrm{Z}^{0} \rightarrow \tilde{\mathrm{h}} \tilde{\mathrm{h}} \mathrm{h}\right) / \Gamma\left(\mathrm{Z}^{0} \rightarrow \mu^{+} \mu^{-}\right)$as a function of $m_{\mathrm{h}} / m_{\mathrm{Z}}$ for model II in the appendix (SU(5) broken with the 75 Higgs). The supergravity parameters are: $A=3, \lambda=0.4, \xi=3$ and $\mu=2 \mathrm{GeV}$. Typical values of slepton and squark masses as well as the standard model prediction for the process $\Sigma \Gamma\left(\mathrm{Z}^{0} \rightarrow \mathrm{h} \nu \bar{\nu}\right) / \Gamma\left(\mathrm{Z}^{0} \rightarrow \mu^{+} \mu^{-}\right)$are included.

parameter $\lambda$ is considered as a free parameter and its value will reflect the structure of the hidden sector of the theory. This hierarchy, $\mu \ll m_{\mathrm{W}}$, translates into $m_{\tilde{\mathrm{h}}} \ll$ $m_{\mathrm{Z}}, m_{\tilde{\mathrm{z}}}$ so that the decay rates $\mathrm{Z} \rightarrow \tilde{\mathrm{z}} \tilde{\mathrm{h}}$, eq. (16), $\tilde{\mathrm{Z}} \rightarrow \mathrm{A} \tilde{\mathrm{h}}$, eq. (10), and $\tilde{\mathrm{z}} \rightarrow \mathrm{h} \tilde{\mathrm{h}}$, eq. (11), are not suppressed by phase space.

The electroweak breaking proceeds as in ref. [14] and we refer the reader there for details. The scalar-neutrino $o^{\star}$ squared mass gets a negative contribution from the $D$-terms, and becomes $m_{\tilde{\nu}}^{2}=|\cos 2 \beta| \mu^{2}$. This means that the scalar-neutrino (actually $\tilde{\nu}_{\tau}$ ) usually becomes the lightest supersymmetric partner and the decay $\tilde{\mathbf{z}} \rightarrow \boldsymbol{\nu} \tilde{\nu}$ is the dominant decay, as assumed to obtain the decay given in eq. (19). The PEP/PETRA bounds [13] for scalar-lepton masses imply $m_{\tilde{\mathrm{q}}}>(65-70) \mathrm{GeV}$ and

* Here we neglect the bottom mass so that we do not make any discrimination among the three generations. 
$m_{\tilde{\mathrm{g}}}>(75-80) \mathrm{GeV}$, which means that the decay $\tilde{\mathbf{z}} \rightarrow \mathrm{q} \overline{\mathrm{q}} \tilde{\mathrm{g}}$ is kinematically suppressed or, most often, forbidden. These bounds on $m_{\tilde{\mathrm{q}}}$ and $m_{\tilde{\mathrm{g}}}$ are consistent with the bounds obtained in ref. [11] based on recent UA1 data.

There are two light Higgs particles in the model: (i) A scalar Higgs boson $h$ that gets most of its mass from radiative corrections à la Coleman-Weinberg [18]: $m_{\mathrm{h}}^{2} \propto \alpha_{3} m_{3 / 2}^{2}$. (ii) A pseudoscalar A (the supersymmetric partner of $\tilde{\mathrm{h}}$ ) whose squared mass is proportional to $\mu m_{3 / 2}$. These masses cannot be very large since the condition $\left\langle\mathrm{H}_{1}\right\rangle \neq 0$ translates into the bound [14] $m_{3 / 2}^{2}<\frac{1}{2} m_{\mathrm{Z}}^{2}$.

In figs. 1 and 2 we show the predictions of model $I$ for the decay rates $\Gamma\left(\mathrm{Z}^{0} \rightarrow \tilde{\mathrm{h}} \tilde{h} \mathrm{~h}\right) / \Gamma\left(\mathrm{Z}^{0} \rightarrow \mu^{+} \mu^{-}\right)$and $\Gamma\left(\mathrm{Z}^{0} \rightarrow \tilde{\mathrm{h}} \tilde{\mathrm{h}} \mathrm{A}\right) / \Gamma\left(\mathrm{Z}^{0} \rightarrow \mu^{+} \mu^{-}\right)$. (In using eq. (19) to obtain these curves, we have used $\Gamma\left(\mathrm{Z}^{0} \rightarrow \mu^{+} \mu^{-}\right) \approx 0.5 \Gamma\left(\mathrm{Z}^{0} \rightarrow \nu \bar{\nu}\right)$.) The parameters of supergravity that we have chosen are: $A\left(M_{\mathrm{X}}\right)=3, B\left(M_{\mathrm{X}}\right)=A\left(M_{\mathrm{X}}\right)-1=2$, $\lambda=0.4, \xi \equiv M_{3}\left(M_{\mathrm{X}}\right) / m_{3 / 2}=1.8$ and $\mu=2 \mathrm{GeV}$. The parameters $A$ and $B$ are the

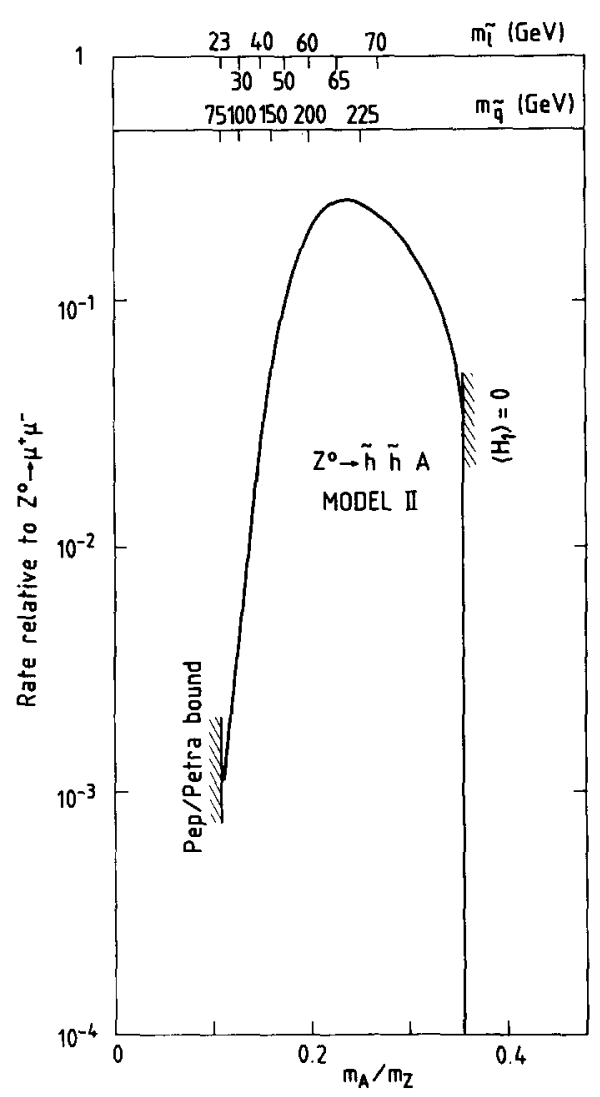

Fig. 4. Decay rate $\Gamma\left(\mathrm{Z}^{0} \rightarrow \tilde{\mathrm{h}} \tilde{\mathrm{h}} \mathrm{A}\right) / \Gamma\left(\mathrm{Z}^{0} \rightarrow \mu^{+} \mu^{-}\right)$as a function of $m_{\mathrm{A}} / m_{\mathrm{Z}}$ for model II. The supergravity parameters are as in fig. 3 . 
usual soft-breaking parameters of supergravity and $\xi$ is the usual gluino to gravitino mass ratio. In fig. 1 we compare with the prediction of the standard model for $\Sigma \Gamma\left(\mathrm{Z}^{0} \rightarrow \mathrm{h} \nu \bar{\nu}\right) / \Gamma\left(\mathrm{Z}^{0} \rightarrow \mu^{+} \mu^{-}\right)$, which is the main background to our proposed supersymmetric source of Higgs bosons. Here, we sum over three generations of massless neutrinos: this ratio is therefore about six times $\Gamma\left(\mathrm{Z}^{0} \rightarrow \mathrm{h} \mu^{+} \mu^{-}\right) / \Gamma\left(\mathrm{Z}^{0} \rightarrow\right.$ $\mu^{+} \mu^{-}$) which was first computed by Bjorken [19]. (Note that $Z^{0} \rightarrow A \nu \bar{\nu}$ does not occur at tree level due to the absence of a ZZA coupling.) In figs. 3 and 4 the corresponding predictions of model II are shown with the choice of supergravity parameters: $A\left(M_{\mathrm{X}}\right)=3, \lambda=0.4, \xi=3$, and $\mu=2 \mathrm{GeV}$. In all cases the decay rates vanish when $\sin 2 \beta \rightarrow 0$ which corresponds to the limit $\left\langle\mathrm{H}_{1}\right\rangle \rightarrow 0$ (i.e. $m_{\mathrm{b}} \rightarrow 0$ ).

\section{References}

[1] H.E. Haber and G.L. Kane, Phys. Reports 117 (1985) 75

[2] J. Ellis, in Proc. Yukon Advanced Study Institute on the Quark structure of matter, ed. N. Isgur, G. Karl and P.J. O'Donnell (World Scientific, Singapore, 1985), p. 256;

R.M. Barnett, in Proc. 13th SLAC Summer Institute on Particle Physics (July 29-August 9, 1985)

[3] E. Witten, Nucl. Phys. B188 (1981) 513;

S. Dimopoulos and H. Georgi, Nucl. Phys. B193 (1981) 150;

N. Sakai, Z. Phys. C11 (1981) 153

[4] K. Inoue, A. Komatsu and S. Takeshita, Prog. Theor. Phys. 67 (1982) 927; (E)70 (1983) 330; 71 (1984) 413

[5] R. Flores and M. Sher, Ann. Phys. (NY) 148 (1983) 95;

H.P. Nilles and M. Nusbaumer, Phys. Lett. 145B (1984) 73;

P. Majumdar and P. Roy, Phys. Rev. D30 (1984) 2432;

M. Drees, M. Gluck and K. Grassie, Phys. Lett. 159B (1985) 32

[6] J.F. Gunion and H.E. Haber, Nucl. Phys. B272 (1986) 1

[7] J.F. Gunion and H.E. Haber, Nucl. Phys. B278 (1986) 449

[8] J. Ellis and G.G. Ross, Phys. Lett. 117B (1982) 397;

J.-M. Frère and G.L. Kane, Nucl. Phys. B223 (1983) 331

[9] M. Quirós, G.L. Kane and H.E. Haber, Nucl. Phys. B273 (1986) 333

[10] H.E. Haber, in Proc. 13th SLAC Summer Institute on Particle Physics (July 29-August 9, 1985)

[11] R.M. Barnett, H.E. Haber and G.L. Kane, Nucl. Phys. B267 (1986) 625

[12] W. Bartel et al., Phys. Lett. 139B (1984) 327

[13] Sau Lan Wu, in Proc. 1985 Conf. of the Division of Particles and Fields of the American Physical Society, Eugene, OR (August 12-15, 1985)

[14] M. Quirós, Nucl. Phys. B271 (1986) 253

[15] M. Levi (UA1 Collaboration), contribution to the Int. Conf. on High-energy physics, Bari, Italy (July 18-24, 1985)

[16] J. Ellis, K. Enqvist, D.V. Nanopoulos and K. Tamvakis, Phys. Lett. 155B (1985) 381

[17] E. Cremmer, S. Ferrara, L. Girardello and A. Van Proeyen, Phys. Lett. 116B (1982) 231; Nucl. Phys. B212 (1983) 413

[18] C. Kounnas, A.B. Lahanas, D.V. Nanopoulos and M. Quirós, Nucl. Phys. B236 (1984) 438

[19] J.D. Bjorken, in Proc. SLAC Summer Institute on Particle Physics (August 2-13, 1976) ed. M.C. Zipf (SLAC-198, 1976) p. 1 